\title{
Comorbidity: Implications for the Importance of Primary Care in 'Case' Management
}

\author{
Barbara Starfield, MD, MPH, FRCGP' \\ Klaus W. Lemke, $P b D^{1}$ \\ Terence Bernbardt, BA \\ Steven S. Foldes, $P b D^{2}$ \\ Christopber B. Forrest, $M D, P b D^{1}$ \\ Jonathan P. Weiner, DrPH \\ 'Department of Health Policy and Manage- \\ ment, Johns Hopkins University Bloomberg \\ School of Public Health, Baltimore, Md \\ ${ }^{2}$ Blue Cross Blue Shield of Minnesota, \\ St. Paul, Minn
}

\begin{abstract}
BACKGROUND Although comorbidity is very common in the population, little is known about the types of health service that are used by people with comorbid conditions.

METHODS Data from claims on the nonelderly were classified by diagnosis and extent of comorbidity, using a case-mix measure known as the Johns Hopkins Adjusted Clinical Groups, to study variation in extent of comorbidity and resource utilization. Visits of patients (adults and children) with 11 conditions were classified as to whether they were to primary care physicians or to other specialists, and whether they involved the chosen condition or other conditions.

RESULTS Comorbidity varied within each diagnosis; resource use depended on the degree of comorbidity rather than the diagnosis. When stratified by degree of comorbidity, the number of visits for comorbid conditions exceeded the number of visits for the index condition in almost all comorbidity groups and for visits to both primary care physicians and to specialists. The number of visits to primary care physicians for both the index condition and for comorbid conditions almost invariably exceeded the number of visits to specialists. These patterns differed only for uncommon conditions in which specialists played a greater role in the care of the condition, but not for comorbid conditions.

CONCLUSIONS In view of the high degree of comorbidity, even in a nonelderly population, single-disease management does not appear promising as a strategy to care for patients. In contrast, the burden is on primary care physicians to provide the majority of care, not only for the target condition but for other conditions. Thus, management in the context of ongoing primary care and oriented more toward patients' overall health care needs appears to be a more promising strategy than care oriented to individual diseases. New paradigms of care that acknowledge actual patterns of comorbidities as well as the need for close coordination between generalists and specialists require support.
\end{abstract}

Ann Fam Med 2003;1:8-14. DOI: 10.1370/afm.1

\section{INTRODUCTION}

Conflicts of interest: None reported

\section{CORRESPONDING AUTHOR}

Barbara Starfield, MD, MPH, FRCGP Johns Hopkins University Bloomberg School of Public Health 624 North Broadway, Room 452 Baltimore, MD 21205 bstarfie@jhsph.edu
$\mathrm{O}$ ne of the challenges resulting from the successes of both preventive and curative health services is the increase in the extent of comorbidity, that is, the co-occurrence of apparently unrelated diseases. Increases in survival would be expected to result in an increase in residual handicap as well as increased vulnerability to additional illnesses. Comorbidity provides new challenges to health care services that have traditionally been focused on individual diseases and with little substantial collaboration between primary care physicians and other specialist physicians. A recent study of the results of such collaboration found only 7 studies. ${ }^{1}$ 
Although the literature on comorbidity is limited, evidence is accumulating that comorbidity is a salient feature among all populations. In 1984, 35\%, 47\%, and $53 \%$ of men of ages 60 to 69,70 to 79 , and 80 years of age and older, respectively, had 2 or more chronic conditions, ${ }^{2}$ with higher levels among women. ${ }^{2,3}$ Of the 90 million Americans with a chronic condition in the early 1990 s, $45 \%$ had more than $1 .^{4}$

Roos et $\mathrm{al}^{5}$ reported that only one third of visits of adult patients with hypertension were for that diagnosis. Clouse and Osterhaus ${ }^{6}$ reported that adults with migraine have $30 \%$ higher costs for conditions unrelated to migraine than patients without migraine. One eighth of the costs of asthma and chronic otitis media and eustachian tube disorders are attributable to diagnosis and treatment of just 1 comorbidity. ${ }^{7}$

The extent of comorbidity increases progressively with age. ${ }^{8}$ However, it is in the young, particularly in children, where unexpected co-occurrence of ill health is most marked. ${ }^{8}$

The impact of comorbidity is considerable. In $1999,65 \%$ of the US elderly had 2 or more types (generally categorized by an organ system involvement or etiology) of chronic conditions. Per capita annual expenditures were $\$ 1154$ for those with 1 type, $\$ 2394$ for those with 2 types, $\$ 4701$ for those with 3, and $\$ 13973$ for those with 4 or more. ${ }^{9}$

Guevara et $\mathrm{al}^{10}$ reported that the prevalence of chronic physical health problems was about $13 \%$ higher in children with ADHD than in other children. The increase in costs with increasing comorbidity was much greater in primary care than in specialty care, and was greater for all categories of use except for mental health services among children with mental health comorbidity.

The purpose of this paper is to elucidate patterns of care for people with comorbidity of varying degrees. In particular, it seeks to examine the extent to which primary care and specialist care play a role in the care of individuals with variable degrees of comorbidity, with the ultimate goal to determine the most appropriate locus of intervention, extending beyond individual visits into periods of time.

\section{METHODS}

\section{Data Source and Study Population}

We conducted a retrospective analysis of administrative claims data of nonelderly patients enrolled in a Midwestern BlueCross BlueShield Point-of-Service (POS) managed care plan, a triple-option POS plan with 3 levels of cost-sharing. The study sample included persons under age 65 who were enrolled for all of
1996 through similar large-group contracts. Of the 322068 enrollees in the plan, 211773 (66\%) met these inclusion criteria for the study sample; of that group, 170447 (80\%) used services in the year: 119 195 adults and 51252 children younger than 18 .

Plan membership files provided data on patient age, sex, and enrollment duration. Every diagnosis recorded on any claim form for the year was included. However, because the plan had a mental health carve-out, most psychosocial diagnoses were not included. Low, medium, and high degrees of morbidity were characterized by the Johns Hopkins Adjusted Clinical Group (ACG) case-mix system, which is described elsewhere. ${ }^{11}$ In brief, the computer program categorizes diagnoses into 32 groups, the diagnoses in each group being similar in type of morbidity. Typical "types" of diagnosis are acute self-limited conditions, likely-to-recur conditions, and chronic stable conditions. (For example, diabetes and hypertension are both in the chronic stable group. Upper respiratory infections are acute self-limited conditions, and sinusitis is a likely-to-recur condition.) No procedures are coded in this case-mix system. Individuals with specific patterns of types of diagnosis are assigned to 1 of 89 mutually exclusive cells (ACGs) that characterize an individual's overall pattern of comorbidity based primarily on the mixture of their diagnosis types. For this study, the 89 ACGs were divided into 3 groups according to the number of types of condition made for each patient over a period of 1 year. The "low" group consisted of people in ACGs with 1 to 3 different types of condition; the "medium" group consisted of people in ACGs with 4 to 5 different types, and the "high" group had people with 6 or more different types of condition diagnosed during the year. Table 1 shows the distribution of patients into the 3 comorbidity groups.

We selected several common and uncommon conditions ("index conditions") and characterized them as to their overall pattern of comorbidity. To examine the impact of burdens of comorbidity on resource consumption, we used the plan-allowed amount for each patient as a proxy for resource consumption. We calculated a health services resource ratio, constituting the average resource use of patients in each of the groups relative to the overall resource use of all patients. The ratio is thus 1.0 for the total population of adult and pediatric users. Ratios greater than 1.0 indicate that a subgroup of patients is more costly compared to the patient population, and subgroups with ratios less than 1.0 are less costly compared to the patient population.

Physician specialty was determined by linking physician names with the American Medical Association (AMA) master file for self-reported specialty. Plan provider files assigned a specialty to physicians whose names were unmatched. Family physicians, general 


\begin{tabular}{|lccccc|}
\hline \multicolumn{5}{l}{ Table 1. Percent Distribution of Comorbidity Groups } & within Age Groups \\
\hline Age Group & Comorbidity & Comorbidity & $\begin{array}{c}\text { Medium } \\
\text { Comorbidity }\end{array}$ & $\begin{array}{c}\text { High } \\
\text { Comorbidity }\end{array}$ & All \\
Adults & 20.6 & 54.3 & 15.1 & 10.0 & 100.0 \\
Children & 17.0 & 65.7 & 13.9 & 3.4 & 100.0 \\
Total & 19.6 & 57.6 & 14.8 & 8.0 & 100.0 \\
\hline
\end{tabular}

groups as well as the health services resources ratio for each disease and comorbidity subgroup and for the user population with comorbidity. Within each disease group, there is a range of degree of comorbidity; patients in higher comorbidity groups have consider-

internists, and general pediatricians were considered primary care physicians. (We repeated the analyses including obstetricians/gynecologists as primary care physicians, with almost identical results.) Physicians who had both primary care and specialist physician designations were considered specialists.

The Johns Hopkins School of Public Health Committee on Human Research deemed the study protocol as exempt from review.

\section{Data Analysis}

We calculated average numbers of visits to outpatient facilities, emergency rooms, and physician practices for persons with the selected disease conditions in each of the 3 comorbidity groups. Primary care physician contacts were separated from other specialist contacts. We used the $t$-test to compare expected visits to generalists and specialists for each condition and level of comorbidity.

\section{RESULTS}

Table 2 presents the distribution of individuals with the selected conditions into each of the 3 comorbidity ably greater resource use than patients in lower comorbidity groups, regardless of the condition. There is no overlap of comorbidity groups for any of the conditions; the greater the comorbidity, the greater the resource use. Moreover, for the most part, differences in resource use are relatively similar for conditions in the same comorbidity category. Although the values in Table 2 are not age-gender adjusted, the figures are similar (within 10 percentage points for distribution and with minor differences for resource ratios) across age groups (0-17, 18-44,45-64) and between females and males.

We examined the frequency of visits to primary care physicians and specialists for the index conditions and for other conditions. Figure 1 shows the pattern of use for adult patients with hypertension. When stratified by group and by visits to primary care physicians and specialist physicians separately, it is apparent that the number of visits for other conditions exceeds the number of visits for hypertension itself in all but the low comorbidity groups. The pattern of greater number of visits for other conditions than for the index condition in all comorbidity groups is found for adults and children with asthma and for adults with ischemic

\section{Table 2. Distribution of Comorbidity Groups within Selected Disease Categories with Health Services Resource Ratios}

\begin{tabular}{|c|c|c|c|c|c|c|c|c|}
\hline \multirow[b]{2}{*}{ Condition } & \multirow[t]{2}{*}{$\begin{array}{l}\text { Prevalence } \\
\text { per } 1000\end{array}$} & \multicolumn{3}{|c|}{$\begin{array}{l}\text { Percent Distribution } \\
\text { of Comorbidity Groups }\end{array}$} & \multicolumn{4}{|c|}{$\begin{array}{c}\text { Health Services Resources Ratio } \\
\text { by Comorbidity Group }\end{array}$} \\
\hline & & Low & Medium & High & Low & Medium & High & All Groups \\
\hline Hypertension & 64.4 & 47.4 & 23.1 & 29.6 & 0.55 & 1.78 & 5.56 & 2.32 \\
\hline Lipoid metabolism disorder & 42.3 & 45.8 & 24.1 & 30.2 & 0.57 & 1.76 & 5.14 & 2.23 \\
\hline Asthma, pediatric & 31.8 & 41.7 & 34.5 & 23.8 & 0.41 & 0.93 & 3.43 & 1.31 \\
\hline Diabetes, adult & 23.0 & 40.4 & 26.4 & 33.2 & 0.62 & 2.22 & 7.13 & 3.21 \\
\hline Asthma, adult & 18.8 & 32.9 & 30.0 & 37.0 & 0.61 & 1.44 & 4.92 & 2.46 \\
\hline Ischemic heart disease & 13.0 & 23.6 & 26.9 & 49.5 & 0.69 & 3.08 & 9.15 & 5.52 \\
\hline Thrombophlebitis & 2.9 & 21.8 & 26.4 & 51.7 & 0.66 & 1.88 & 7.95 & 4.75 \\
\hline Congestive heart failure & 2.0 & 14.9 & 21.5 & 63.6 & 0.68 & 3.12 & 12.96 & 9.01 \\
\hline Multiple sclerosis & 2.0 & 34.6 & 28.2 & 37.2 & 0.67 & 2.70 & 7.48 & 3.78 \\
\hline Diabetes, pediatric & 1.9 & 42.5 & 35.0 & 22.5 & 0.46 & 1.64 & 6.54 & 2.24 \\
\hline Chronic liver disease & 1.3 & 18.1 & 21.8 & 60.1 & 0.68 & 3.03 & 10.44 & 7.06 \\
\hline
\end{tabular}




\section{Figure 1. Visits to Generalist and Specialist Physicians for Adults with Hypertension}

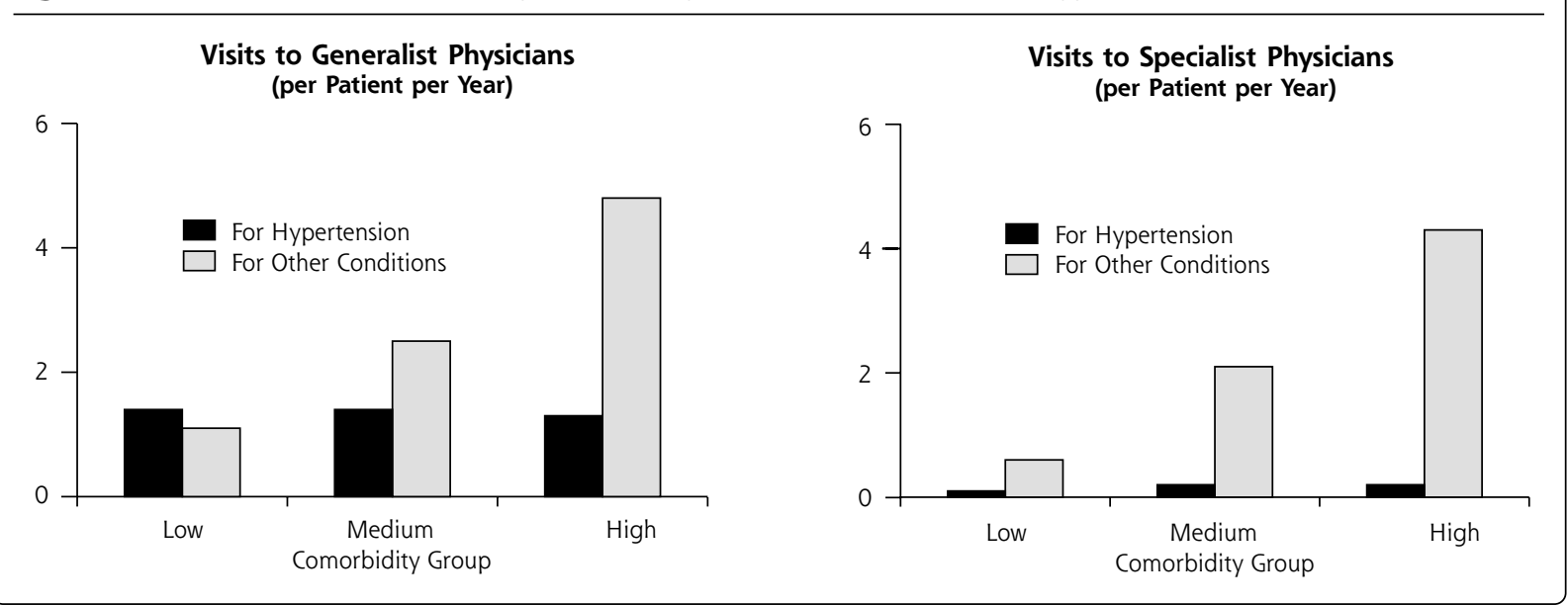

\section{Figure 2. Visits to Generalist and Specialist Physicians for Children with Diabetes}

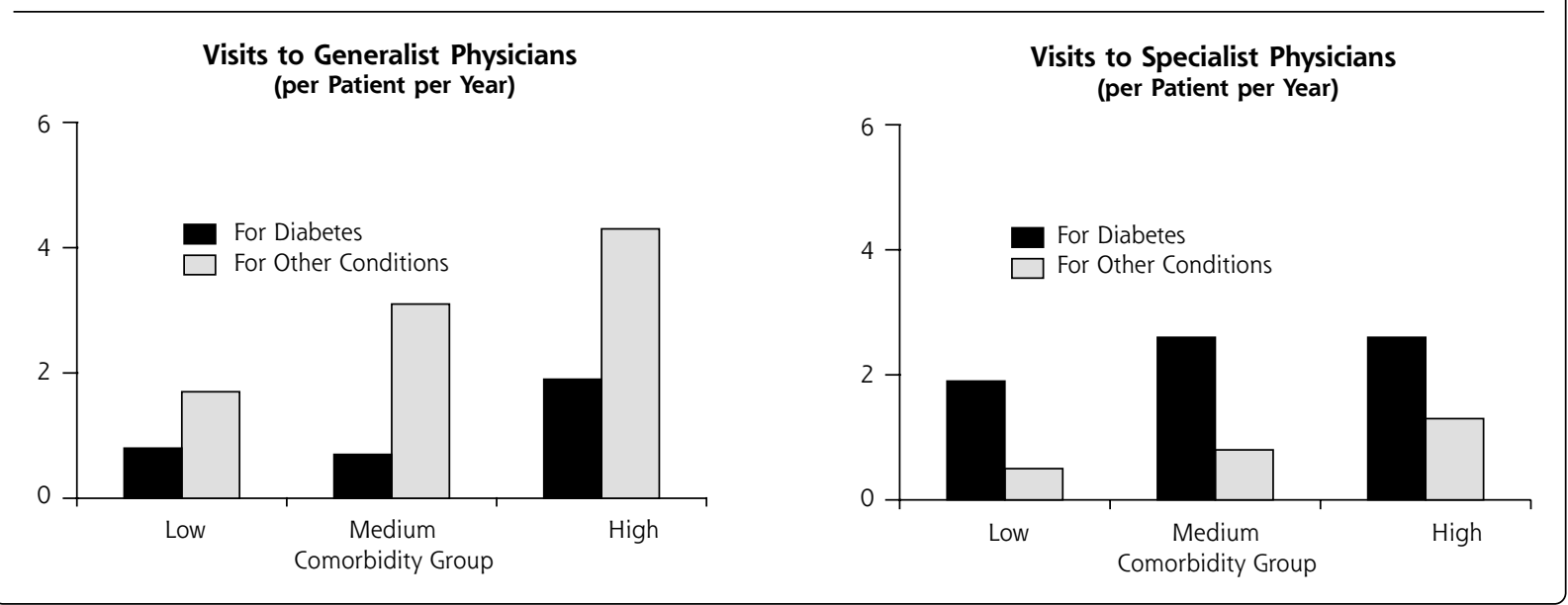

heart disease, thrombophlebitis, chronic liver disease, congestive heart failure, or lipoid metabolism disorder. The pattern is similar for adults with diabetes, except that patients in the low comorbidity group made an equal number of visits to specialists for the condition and for other conditions. The pattern diverges only for the uncommon condition in childhood: diabetes mellitus (Figure 2). In this case, patients visited specialists more frequently for the condition than for other conditions.

Table 3 shows that the number of visits to primary care physicians exceeds that to specialists, for both the condition and for comorbid conditions, in most groups to a statistically significant degree. The exceptions are in the less common conditions at the bottom of the table wherein the number of visits to specialists for the condition itself was more likely to be greater than the number of visits to primary care physicians at all levels of comorbidity. Thus, for patients with all of the con- ditions studied, the number of visits to generalist physicians for the selected diagnosis exceeds the number of visits to specialists, at all degrees of comorbidity, except in people with some uncommon conditions. Although this could conceivably be interpreted as indicating that primary care physicians are less efficient than other specialists, it seems much more likely that the greater number of visits is a response to patient needs of different types.

\section{DISCUSSION}

In this large managed care organization, which provides access to specialists both on referral or by selfreferral, primary care providers are the major providers of care both for the index condition and for other conditions, and for patients with all degrees of comorbidity, except for uncommon chronic conditions. In the case of common conditions, even people with high 
levels of comorbidity see specialists less often than primary care physicians for both the index condition and for other conditions. The number of visits, both to primary care physicians and to specialists, is highly related to the degree of comorbidity. In the case of uncommon conditions, specialists are more likely to be the locus of care than generalists for the index condition (although not for comorbid conditions); previous research has shown that primary care physicians are more likely to refer patients with uncommon conditions than patients with common conditions. ${ }^{12}$

Kuhlthau et $\mathrm{al}^{13}$ reported similar findings for chronically ill children on Medicaid. In their study, children with chronic health conditions, especially those with more common conditions, used generalist care much more commonly than subspecialist care. The study also showed that the likelihood of a specialist visit increased with increasing case-mix, using the same ACG case-mix system as applied in our study.

One possible limitation of our study is a result of the fact that all subjects were members of an $\mathrm{HMO}$ health plan. As such, it is possible that patients in our data set may have been less likely to use healthcare services overall, and specialist services in particular, than if they had been in a conventional fee-for-service, open access indemnity plan. Specialist utilization may be higher where there are no gate-keeping restrictions, although recent research suggests this is not the case. ${ }^{14,15}$ On the other hand, given that most of our study group received their care from well-organized multi-specialty groups and networks, this could have led to increased access to specialists. Furthermore, health care systems in which visits to specialists are controlled to some degree by primary care physicians are commonplace in some countries and increasingly the case in others.

Restriction of the study population to those under age 65 underestimates the extent of comorbidity in the population as a whole. Although our results can be expected to pertain to other similar under-65 insured populations, these findings will need to be replicated elsewhere, including among the elderly, before they can be widely generalized.

A third limitation is that all possible diagnoses that were made in visits may not have been recorded, thus underestimating the extent of comorbidity. Only 1 in 10 visits had more than 1 diagnosis recorded. We were thus unable to examine the extent to which comorbidity is actually addressed in physician visits. We cannot confidently conclude either that comorbidity is addressed or not addressed either by primary care physicians or specialists. It is also possible that some of the "other" conditions were pathophysiologically related to the selected diagnosis; if so, the relative dearth of visits to specialists for these other conditions is all the more striking.

A fourth limitation is the exclusion of claims for psychosocial services. This would also reduce the extent of measured comorbidity. A fifth limitation derives from the relative dearth of low-income people in a midwestern $\mathrm{HMO}_{\text {; }}$ such populations would be expected to have greater morbidity and perhaps differ-

Table 3. Mean Numbers of Visits with Generalist and Specialist Physicians for Index Condition and for Other Conditions by Comorbidity Group

\begin{tabular}{|c|c|c|c|c|c|c|}
\hline \multirow[b]{3}{*}{ Condition } & \multirow{2}{*}{\multicolumn{3}{|c|}{$\begin{array}{c}\text { Number of Visits for Index Condition } \\
\text { (Primary Care I Specialist) } \\
\text { Comorbidity Group }\end{array}$}} & \multirow{2}{*}{\multicolumn{3}{|c|}{$\begin{array}{c}\text { Number of Visits for Other Conditions } \\
\text { (Primary Care I Specialist) } \\
\text { Comorbidity Group }\end{array}$}} \\
\hline & & & & & & \\
\hline & Low & Medium & High & Low & Medium & High \\
\hline Hypertension & $1.38 / 0.12 * * *$ & $1.38 / 0.17 * * *$ & $1.25 / 0.20 * * *$ & $1.07 / 0.61 * * *$ & $2.51 / 2.11 * * *$ & $4.79 / 4.31 * * *$ \\
\hline Lipoid metabolism disorders & $1.03 / 0.15^{* * *}$ & $1.01 / 0.19 * * *$ & $0.86 / 0.18 * * *$ & $1.19 / 0.59 * * *$ & $2.62 / 1.83 * * *$ & $4.78 / 3.87 * * *$ \\
\hline Asthma, children & $1.05 / 0.26 * * *$ & $1.16 / 0.28^{* * *}$ & $1.05 / 0.54 * * *$ & $2.29 / 0.57 * * *$ & $4.67 / 1.35^{* * *}$ & $8.19 / 3.25^{* * *}$ \\
\hline Diabetes, adults & $1.50 / 0.66^{* * *}$ & $1.62 / 0.90 * * *$ & $1.84 / 1.32 * * *$ & $1.14 / 0.64 * * *$ & $2.31 / 1.70 * * *$ & $4.52 / 4.43$ \\
\hline Asthma, adults & $0.91 / 0.59 * * *$ & $0.83 / 0.64 *$ & $0.94 / 1.03$ & $1.56 / 0.80 * * *$ & $3.73 / 2.09 * * *$ & $6.42 / 4.78^{* * *}$ \\
\hline Ischemic heart disease & $0.96 / 0.40 * * *$ & $0.87 / 0.59 * * *$ & $0.96 / 0.70 * * *$ & $1.30 / 0.66 * * *$ & $2.54 / 1.62 * * *$ & $5.00 / 3.92 * * *$ \\
\hline Thrombophlebitis & $1.01 / 0.17 * * *$ & $1.11 / 0.21 * * *$ & $1.26 / 0.28^{* * *}$ & $1.25 / 0.69 * *$ & $2.67 / 1.91^{*}$ & $5.24 / 5.24$ \\
\hline Congestive heart failure & $0.93 / 0.49$ & $1.17 / 0.49 *$ & $0.96 / 0.44 * *$ & $2.44 / 1.29 *$ & $3.74 / 1.72 * * *$ & $6.75 / 6.90$ \\
\hline Multiple sclerosis & $0.89 / 1.51 * *$ & $0.54 / 1.73 * * *$ & $0.99 / 2.19 * * *$ & $1.00 / 0.59 * *$ & $2.49 / 2.27$ & $4.27 / 4.29$ \\
\hline Diabetes, children & $0.80 / 1.92 * *$ & $0.74 / 2.57 * * *$ & $1.85 / 2.63^{\mathrm{a}}$ & $1.71 / 0.45^{* * *}$ & $3.07 / 0.81 * * *$ & $4.26 / 1.33 * * * a$ \\
\hline Chronic liver disease & $0.71 / 0.83$ & $0.33 / 0.86$ & $0.68 / 0.51$ & $1.29 / 0.97$ & $2.83 / 2.24$ & $5.51 / 5.08$ \\
\hline $\begin{array}{l}\text { Unless otherwise indicated, preval } \\
\text { a. Fewer than } 30 \text { cases in category } \\
\begin{array}{rl}* P & <.05 \text { for comparing mean } \\
* * & <.01 \\
* * * & P<.001\end{array}\end{array}$ & ces are for adults. & norbidity Groups a & mean number of & $\begin{array}{l}\text { Clinical Groups (A } \\
\text { ts. }\end{array}$ & & \\
\hline
\end{tabular}


ent patterns of use of generalists and specialists. A sixth potential limitation is absence of information on relative quality of care or outcomes of care.

Our findings have implications concerning the quality of care for people with comorbidities and the role of primary care physicians in case management. With regard to quality of care, the findings do not bear on which type of physician (generalist or specialist) provides the highest quality of care for specific conditions. Most studies that compare generalists and specialists conclude that quality of care provided by specialists is better in their area of special interest, using conventional "HEDIS-type" indicators of quality of care (http://www.ncqa.org) such as the performance of indicated preventive procedures, the performance of indicated laboratory tests for monitoring disease status, and the prescribing of indicated medications. ${ }^{16}$ The few studies planned and executed by primary care physicians conclude that quality of care is the same or that primary care is better. ${ }^{17-19}$ This would suggest possible differences in conceptualization of appropriate "outcomes" by the 2 types of physician, specialists being more concerned with specific disease-related measures. Few studies have examined generic outcomes, in terms of health status, or quality of care other than for particular conditions under study, although our evidence indicates that comorbidity engenders more visits to both generalists and specialists than does the index condition. If it is patient health (rather than disease processes or outcomes) that is of interest as the proper focus of health services, a broader view of quality of care provided to patients is in order.

Our findings support the premise that new or expanded roles for primary care physicians and their relationships with specialists are needed. The high salience of comorbidity makes it unlikely that management of a patient visit by visit, with each visit focused primarily on 1 condition, can provide effective care from the vantage of the patient, even in patients with bona fide common conditions. In the case of common conditions, the large proportion of visits to generalist physicians rather than to disease-oriented specialists and the frequency of such visits for both the specific condition and for comorbid conditions suggests a major role for primary care physicians, operating in a patient ("case") management mode, with a strong imperative for appropriate consultation with specialists. ${ }^{20}$ For uncommon conditions, the imperative is to develop expanded and more effective coordination of care between primary care physicians and specialists. Communication between primary care physicians and specialists occurs in only $50 \%$ to $85 \%$ (depending on the types of communication) of all initial referrals from primary care physicians. ${ }^{21}$ Communication concerning visits that occur by self referral, or by request of the specialist for return by the patient, must surely be lower than this; the literature ${ }^{22}$ indicates very poor communication overall.

These concerns suggest the need to seriously reexamine the nature of the relationship between primary care physicians and specialists. In some areas, there are efforts to reduce the extent to which the patient visits the specialist directly. Instead, specialists make visits to primary care offices to see patients or groups of patients, in this way both improving the knowledge of the primary care physician and enhancing convenience for the patient. ${ }^{22}$ Such a process should also serve to make specialists more aware of comorbidity among patients with diseases not in their area of particular interest, and perhaps to highlight the need to modify disease-oriented guidelines, most of which have been developed from studies on patients without other concurrent illnesses.

From a health-plan perspective, management should include a continuum-based approach to healthcare delivery that proactively identifies populations and individual plan members with, or at risk for, medical conditions, regardless of what the conditions are. The considerable prevalence of patients with comorbidities suggests that selecting patients for these programs based on a single index condition may fail to identify those patients with complex needs who may benefit most from improved coordination of care. At the level of a health plan, so-called "carve outs" for selected conditions (mental health being only the most common) present a potential threat to the plan's ability to help physicians bridge the gaps in patients with comorbidities.

Some forms of patient-oriented "case" management (eg, the Chronic Care Model or CCM) are specifically designed to enhance coordination of care, on the grounds that the common characteristics of successful care for major chronic diseases as well as for preventive activities provide strong arguments for care to be coordinated by primary care physicians. ${ }^{23,24}$ These programs focus on 4 concurrent strategies: self-management support; practice teams to achieve clinical and behavioral management; disease support; and a well-designed clinical information system. ${ }^{25}$

The recognition of the large extent of comorbidity in adults, children and among patients with multiple types of illness has implications for the way in which health care is organized. If for no other reason, comorbidity as a common feature of illness makes a compelling case for maintaining a strong primary care basis for any patient care system. It also provides an impetus for rethinking conventional modes of practice and the nature of relationships between primary care physi- 
cians and their specialist counterparts. As the population's needs evolve, so too must systems of care and the paradigms upon which they are built.

To read commentaries or to post a response to this article, see the online version at http://www.annfammed.org/cgi/content/full/1/1/8.

Key words: Comorbidity, Primary Care, Case-Mix, Case Management, Adjusted Clinical Groups.

Received September 24, 2002; Accepted December 31, 2002.

\section{Financial support}

This work was supported in part by Grant No. 6 U30 CS 00189-05 S1 R1 of the Bureau of Primary Health Care, Health Resources and Services Administration, Department of Health and Human Services, by the Primary Care Policy Center for the Underserved at Johns Hopkins University, by the Johns Hopkins University's ACG Case-Mix Research and Development Fund, and by Grant No. 36484 from the Robert Wood Johnson Foundation.

\section{Disclaimer}

The Johns Hopkins University has copyrighted software based on the ACG case-mix system applied in this study. Royalties are paid to the university when this software is used by insurance plans and commercial organizations.

\section{References}

1. Mitchell G, Del Mar C, Francis D. Does primary medical practitioner involvement with a specialist team improve patient outcomes? A systematic review. Br J Gen Pract 2002;52:934-939.

2. Guralnik JM, LaCroix A, Evertt D. Aging in the eighties: the prevalence of co-morbidity and its association with disability. Washington, DC: DHHS (NCHS); 1989.

3. Bierman AS, Clancy CM. Women's health, chronic disease, and disease management: new words and old music? Womens Health Issues 1999;9:2-17.

4. Hoffman C, Rice D, Sung HY. Persons with chronic conditions. Their prevalence and costs. JAMA 1996;276:1473-1479.

5. Roos NP, Carriere KC, Friesen D. Factors influencing the frequency of visits by hypertensive patients to primary care physicians in Winnipeg. Can Med Assoc J 1998;159:777-783.

6. Clouse JC, Osterhaus JT. Healthcare resource use and costs associated with migraine in a managed healthcare setting. Ann Pharmacother $1994 ; 28: 659-664$
7. Ray NF, Baraniuk JN, Thamer M, et al. Healthcare expenditures for sinusitis in 1996: contributions of asthma, rhinitis, and other airway disorders. J Allergy Clin Immunol 1999;103:408-414.

8. van den Akker M, Buntinx F, Metsemakers JF, Roos S, Knottnerus JA. Multimorbidity in general practice: prevalence, incidence, and determinants of co-occurring chronic and recurrent diseases. J Clin Epidemiol 1998:51:367-375.

9. Wolff JL, Starfield B, Anderson G. Prevalence, expenditures, and complications of multiple chronic conditions in the elderly. Arch Intern Med 2002;162:2269-2276.

10. Guevara J, Lozano P, Wickizer T, Mell L, Gephart H. Utilization and cost of health care services for children with attention-deficit/hyperactivity disorder. Pediatrics 2001;108:71-78

11. Health Services Research and Development Center, Johns Hopkins University. The Johns Hopkins ACG Case-Mix System Documentation Manual. Available at http://www.acg.jhsph.edu. Accessed April 15, 2003.

12. Forrest $C B$, Reid RJ. Prevalence of health problems and primary care physicians' specialty referral decisions. J Fam Pract 2001;50:427-432.

13. Kuhlthau K, Ferris TG, Beal AC, Gortmaker SL, Perrin JM. Who cares for medicaid-enrolled children with chronic conditions? Pediatrics 2001;108:906-912.

14. Forrest $C B$, Weiner JP, Fowles J, et al. Self-referral in point-of-service health plans. JAMA 2001;285:2223-2231.

15. Ferris TG, Chang Y, Blumenthal D, Pearson SD. Leaving gatekeeping behind-effects of opening access to specialists for adults in a health maintenance organization. N Engl J Med 2001;345:1312-1317.

16. Harrold LR, Field TS, Gurwitz JH. Knowledge, patterns of care, and outcomes of care for generalists and specialists. J Gen Intern Med 1999;14:499-511.

17. Donohoe MT. Comparing generalist and specialty care: discrepancies, deficiencies, and excesses. Arch Intern Med 1998;158:1596-1608.

18. Grumbach K, Selby JV, Schmittdiel JA, Quesenberry CP Jr. Quality of primary care practice in a large HMO according to physician specialty. Health Serv Res 1999;34:485-502.

19. Greenfield S, Rogers W, Mangotich M, Carney MF, Tarlov AR. Outcomes of patients with hypertension and non-insulin dependent diabetes mellitus treated by different systems and specialties. Results from the medical outcomes study. JAMA 1995;274:1436-1444.

20. Bodenheimer T. Disease management-promises and pitfalls. $N$ Engl J Med 1999;340:1202-1205.

21. Forrest CB, Glade GB, Starfield B, Baker AE, Kang M, Reid RJ. Gatekeeping and referral of children and adolescents to specialty care. Pediatrics 1999;104:28-34.

22. Starfield B. Primary care: balancing health needs, services, and technology. New York, NY: Oxford University Press; 1998.

23. Glasgow RE, Orleans $C T$, Wagner EH. Does the chronic care model serve also as a template for improving prevention? Milbank $Q$ 2001:79:579-612, iv-v.

24. Bodenheimer T, Wagner EH, Grumbach K. Improving primary care for patients with chronic illness. JAMA 2002;288:1775-1779.

25. Wagner EH, Glasgow RE, Davis C, et al. Quality improvement in chronic illness care: a collaborative approach. Jt Comm J Qual Improv 2001;27:63-80. 